\title{
Vascular Malformations of the Brain
}

\author{
Haley Guerin* \\ High School Student and Neurology Enthusiast, USA
}

Submission: March 17, 2017; Published: May 04, 2017

*Corresponding author: Haley Guerin, High School Student, Neurology Enthusiast, Cincinnati, Ohio, USA, Email: hguerin222@gmail.com

\begin{abstract}
Vascular malformations of the brain are complex in nature and may be classified into various subcategories inclusive of arteriovenous malformations, venous angiomas, angiographically occult vascular malformations, dural AVMs, vein of Galen malformations, and carotidcavernous fistulas Symptoms, treatments, behaviors, and fatality levels in respect to surgery vary in correlation with the essence of the vascular malformation being considered. Vascular malformations are not only unique in their disposition, but also in their ability to involve multiple areas of medicine. While such malformations only affect a small portion of the population, they are significant to the general understanding of neurosurgery as well as vascular medicine.
\end{abstract}

\section{Introduction and Historical Report}

Vascular malformations of the brain are greatly disruptive of the process of transporting oxygen-rich blood to the brain and returning it to the lungs and heart [1-3]. This is due to the invasive nature of vascular malformations as an interconnected group of abnormal blood vessels that are otherwise instrumental in linking arteries and veins in the brain. Arterial blood flows directly into specific veins that lack regular interposed capillary beds. Some are congenital lesions that increase in size from birth and may occur on the spine as well. During adult years they are often high-pressure lesions with a well circumscribed nidus. Astrovenial vascular malformations are often pial, combined, periventricular, or sub cortical. Vascular malformations of the brain may be classified based on severity, frequency, causes, and symptoms.

It is crucial to understand which demographics are most susceptible to vascular malformations of the brain in order to increase awareness. It is extremely rare to experience an interaction with a patient who has a vascular malformation of the brain because it has only been reported in less than one percent of the population. This disorder is not generally biased towards a specified gender, race, nationality, or age. In contrast, most cases reported have included individuals between the ages of ten and forty years of age, yet such is a considerably vague range. In addition to this contradicting claim, a few variables remain that allow for bias fluctuation. For example, MexicanAmericans are most likely to inherit cavernous malformations while males are more susceptible than females to arteriovenous malformations.

\section{Etiology of Vascular Malformations}

Many vascular malformations of the brain are the results of genetic inheritance, but an important percentage of the patient population with vascular malformations of the brain develop from trauma or other neurologic issues. Genetic cases include that of cavernous hemangiomas and telangiectasias. In these specific cases, they exist as an autosomal dominant trait that may be inherited from either parent and as the result of a mutation. The gene map locus related to cavernous hemangiomas is locus 7q11.2-q21. Locus 9q34.1 is related to telangiectasias. The possibilities of genetic engineering have a great potential to eliminate this type of disorder, yet remain as a highly controversial area of medicine. Similarly, in the cases of arteriovenous malformations of the brain, the malformation is congenital and cannot easily be avoided. However in other cases, the vascular malformations may develop at a later stage in the individual's life. Dural AVMs of the transverse-sigmoid sinus junction are not congenital. They are identified by lesions that often develop from chronic infection or trauma. The lesions are a product of collateral revascularization that may lead to thrombosis of a venous sinus. In general terms, a single lesion indicates a sporadic etiology while multiple lesions suggest genetic responsibility. The etiology of vascular malformations has the ability to suggest certain behaviors of the specific malformation as well as possible treatment options [4-6].

\section{Symptoms and Diagnosis}

Symptoms of vascular malformations of the brain are dependent upon the size, type, and location of the malformation in 
the brain. Strokes are a popular indicator of these malformations, but may also suggest other neurological disorders. In this case, strokes are classified as hemorrhagic. When thin blood vessel walls of the vascular malformation experience bleeding, a stroke is likely to occur and induce nausea, headaches, vomiting, and a loss of consciousness. Brain imaging technology allows for stroke testing. However, it is essential to note that brain scans may not demonstrate bleeding of the brain due to fluctuations in blood vessel pressures. In some circumstances, the bleeding is so minimal that no symptoms are observed. Bleeding in the brain is a serious medical emergency and often leads to fatality if unnoticed [7-10].

In addition to the previous indication of a vascular malformation, epileptic seizures are also a potential result of vascular malformations of the brain. Over activity of electronic impulses and vascular malformations pressing on the brain can result in seizures. A significant exception to this are vascular malformations centered around the brainstem and cerebellum which will not result in seizures. The risk of developing epilepsy as a result of the formation of an AMV is merely a one percent risk. Cavernous malformations also contain only a one percent risk of developing epilepsy as a result of the formation of an AMV.

A more common symptom of vascular malformations is headaches. Headaches are also the result of several other possible disorders, which makes the relationship between vascular malformations and headaches indefinite. Headaches that are related to vascular malformations of the brain are often severe and resemble a migraine in nature. The pain associate with the headache may not occur where the malformation is located. These types of headaches are the result of discomfort associated with pain fibers in the dural membrane and suggest the possibility of a brain hemorrhage. The complex interconnected network of arteries, capillaries, veins, tissues, and organs allow for disorders of one source to result in several other directly related issues and symptoms, creating a network of related problems. Visual issues that result from vascular malformations of the brain include blurred vision, double vision, or even the loss of vision. Swelling of the eye socket and redness may also occur.

Specific noises in the head identified as bruits or pulsatile tinnitus may also indicate the presence of vascular malformations. Bruits occur when dural arteriovenous vascular malformations result in a noise in the patient's head as a response to uncontrolled blood flowing through the head. A stethoscope may be used to listen to the bruit. If a bruit is heard as a ringing sound that correlates with the rhythm of the patient's pulse then it is classified as pulsatile tinnitus.

Aneurysms have a seven percent chance of occurring in patients with AVMs and thus may indicate the possibility of a vascular malformation. A majority of these aneurysms are located on major arteries. The classifications of aneurysms due to AVMs include I, IA, II, III, and IV. They are primarily classified based on the location of the aneurysm. When treating aneurysms as well as tandem AVMs it may be difficult to understand which one to treat first. The symptomatic one is treated first, but in some cases both may be treated during the same operation under the appropriate circumstances. The aneurysm is more likely to bleed and sixty six percent of aneurysms related to AVMs will revert back after eliminating the AVM. However, other aneurysms may increase in size as a result of removing the arteriovenous vascular malformation.

The final symptoms of vascular malformations in relation to the brain that will be discussed in this article are memory loss, anxiety, and depression. Memory loss and issues associated with attention and concentration are the results of variations in the flow of blood in the brain. Anxiety and depression are not directly caused by vascular malformations of the brain, rather may result because of the emotional instability a patient may experience when confronted with neurological disorder.

\section{Treatment options for arteriovenous vascular malformations}

There are various options for treating arteriovenous vascular malformations specific to the case of the patient with the malformation as well as the behavior of the AVM itself. The primary option is to surgically remove the AVM. Surgery will remove the risk of bleeding in addition to decreasing seizures. However, surgery is invasive and not necessarily cost effective.

A second option to treat arteriovenous vascular malformations is radiation treatment. Radiation treatment is only effective in a maximum of twenty percent of cases. A type of radiation treatment that is used for deep AMVs contains a two and a half to three centimeter nidus and is called stereotactic radiosurgery. It is done as an outpatient treatment and is noninvasive. There is no definite recovery time period for this type of treatment, but there is a gradual decrease in AVM flow. In contrast, it takes an extensive period of time to work, typically one to three years during which the risk of bleeding is still present. Endovascular techniques that are instrumental in treating arteriovenous vascular malformations often feature the insertion of catheters into cerebral vessels that nurture the AVM. When the catheter contacts the nidus, glue material is injected to obstruct areas of the arteriovenous malformation or the entirety of the malformation. This procedure replaces surgery, but normally cannot eliminate AVMs by itself and may demand several additional procedures.

\section{Postoperative deterioration of AVMs}

When examining the postoperative state of a patient there are four particular signs of deterioration to be aware of. The first is normal perfusion pressure breakthrough. It is identified by tissue hemorrhage or possible swelling and is suspect to be due to the loss of auto regulation, yet this theory is debatable. According to this theory, arteries that supply cerebral arteriovenous vascular malformations lose the ability to autoregulate pressure. 
Excessive blood pressure in the arteries can cause tissue to bleed. Risk of perfusion pressure breakthrough may be reduced by the introduction of pre-operative medicines. Occlusive hyperemia is generally caused by delayed thrombosis of either dural sinus or draining vein. It may lead to bleeding in the brain or even neurological deficits that are secondary to venous hypertension. Rebleeding from a retained nidus of arteriovenous vascular malformation may also occur in addition to possible seizures.

\section{Venous angiomas}

Venous angiomas is often referred to as venous malformation or venous anomaly. It is the most common of all intravascular cranial malformations, yet is only relevant to two and a half percent of the population. This type of malformation is primarily identified by its enlarged groupings of veins that receive drainage from a different area of the brain. The veins possess a radial arrangement in which they intersect on an enlarged central venous trunk that drains into venous systems. On an angiography it will appear in a starburst-like pattern. Seizures and hemorrhages are rare in the case of venous angiomas. Unfortunately, the presence of the intervening brain causes treatment to be discouraged. Neural parenchyma is located between the vessels. It is most frequent within the regions supplied by the middle cerebral artery or in the vein of Galen region of the brain. Surgery is only performed when bleeding is reported or intractable seizures that ascribe to the lesion are reported. As mentioned previously, treatment is rare.

\section{Angiographically occult vascular malformations}

Angiographically occult vascular malformations are cerebrovascular malformations that may not be shown on a technically adequate cerebral angiography. Various lesions contain patent vessels that are large in size at surgery. There are several reasons why a vascular lesion may not be identified angiographically. The effect of hemorrhage on the lesion, bleeding of the lesion, a clot compressing the lesion, poor flow, the size of abnormal vessels, and late filling that requires late angiographic films. There are different classifications of angiographically occult vascular malformations that include arteriovenous malformations, venous angiomas, cavernous malformations, and capillary telangiectasia. Osler-WeberRendu syndrome is commonly associated with angiographically occult vascular malformations. This syndrome is an autosomal genetic disorder that is extremely rare. It is related to capillary telangiectasia, and thus is also referred to as hereditary hemorrhagic telangiectasia. This syndrome causes patients to be prone to pulmonary arteriovenous fistulas as well as paradoxical cerebral embolism that may lead to embolic stroke and cerebral abscess formations. Surgery is the most common treatment method for angiographically occult vascular malformations.

\section{Cavernous malformation}

Cavernous malformations are groups of small blood vessels and thin walled blood vessels often located on the brain. This type of malformation may be inherited or sporadic. Seizures may indicate the possibility of this type of malformation and are more likely to appear on a MRI or CT. It is also recognized as angioma, cavernoma, cavernous angioma, or cavernous hemangioma. Cavernous malformations are typically one to five centimeters in size and often do not have intervening neural parenchyma. They also do not typically have large feeding arteries or large draining veins. Lesions have the potential to worsen or heal according to the behavior of the lesion and the circumstance. Hemorrhage is generally not extreme and may not always lead to an increased risk in future bleeding. Surgery may be appropriate when lesions have focal deficits, symptomatic hemorrhage, or seizures are reported. Stereotactic radiosurgery is a possible treatment, but outcomes are not well established.

\section{Dural AVMs}

Dural AVMs are also called dural arteriovenous fistula and are classified based on the feeding arteries that allow for them to be further sub classified as pure pial, mixed pial and dural, or pure dural. Dural AVMs of the transverse-sigmoid sinus junction are not congenital, but rather are classified by the nature of lesions that often develop from chronic infection or trauma. The lesions are a product of collateral revascularization that may lead to thrombosis of a venous sinus. DAVMs are often located next to dural venous sinuses with the epicenter generally located at the intersection of the transverse and sigmoid sinuses. However, dural arteriovenous fistulas may also appear in the area of the tentorium. Dural AVMs constitute ten to fifteen percent of all intracranial AVMs and are most prevalent in middle aged women. Common symptoms are inclusive of headaches, papilledema, visual impairment, occipital bruit, and pulsatile tinnitus. In contrast, some cases of dural arteriovenous fistulas are asymptomatic. Popular surgery options are stereotactic radiosurgery, surgery, and endovascular embolization with each having specific associated risks and benefits depending on the circumstance of the patient.

\section{Vein of galen malformation}

The great cerebral vein of galen is located beneath the cerebral hemispheres and drains certain regions of the brain into the sinuses of the posterior cerebral fossa. Thus, the vein of galen malformations are often classified based on the location of the fistula as a pure internal fistulae, fistula between thalamoperforator and the vein of galen, mixed form, and plexiform AVMs. Such malformations are most likely to form in newborns that have congestive heart failure as a result of high blood flow in addition to a cranial bruit. The patient is at risk of hydrocephalus when a vein of galen malformation is present because of increased venous pressure or the obstruction of the sylvian aqueduct by the vein of galen that has increased in size. If the VOG malformation is untreated, fatalities are likely to occur in respect to infants. However, treatment options are severely restricted due to the general poor medical condition of the patient. Embolization of the central feeding arteries is an 
option for treatment, yet may not produce satisfactory results. Neurosurgical excision is usually a more beneficial option in the case of reported hydrocephalus.

\section{Carotid-Cavernous fistula}

A carotid cavernous fistula is characterized by an abnormal connection between the neck artery and veins located behind the eye that are auxiliary in the functionality of the circulatory system. Carotid cavernous fistulas are classified as post-traumatic or spontaneous. A post-traumatic CCF occurs in less than one percent of patients that are affected by craniocerebral trauma. These types of CCFs may also be present after percutaneous trigeminal rhizotomy. Spontaneous carotid cavernous fistulas may be further subdivided into four main groups. These groups include high-flow shunts placed between the internal carotid artery and cavernous sinus, dural shunts identified by low flow placed between meningeal branches of the cavernous sinus and internal carotid, dural shunts located between meningeal branches of specifically the external carotid artery and the cavernous sinus, as well as dural shunts prevalent between meningeal branches of both the internal and external carotid artery and cavernous sinus. Treatment is favorable because it may decrease diplopia in addition to increasing visual quality from its previous state. Surgery is recommended under the circumstance in which high flow lesions or lesions with progressive visual deterioration are present. Balloon embolization is often beneficial because it has the potential to reduce flow entirely or to a satisfactory state.

\section{Conclusion}

The study of vascular malformations will continue to be instrumental in the contemporary understanding of neurological deficits. Such malformations exist in a convoluted form and require extensive research in order to understand the subject in its entirety. As technological advancements directly influence the field of medicine, an increase in opportunities to study vascular malformations will arise. It is essential to be aware of operative and non-operative circumstances when treating vascular malformations in order to create the most beneficial and statistically proven quality of treatment for the patient. The defining characteristics of specific classifications of vascular malformations dictate the epidemiology, key features, treatment, and behavior of the malformation. The medical perception of vascular malformations is constantly evolving as research progresses and will progressively influence the study of neurosurgery.

\section{References}

1. Greenberg, Mark S (1997) Handbook of Neurosurgery. Greenberg Graphics, Lakeland, UK.

2. Smith $A B$ (2012) Vascular Malformations of the Brain: Radiologic and Pathologic Correlation. J Am Osteopath Coll Radiol 1(1): 10-21.

3. NORD (2017) Vascular Malformations of the Brain. National Organization for Rare Diseases. USA.

4. Brouillard, Pascal, Miikka Vikkula (2007) Genetic Causes of Vascular Malformations. Human Molecular Genetics 16(2): R140-R149.

5. Oxford Academy (2017) Human Molecular Genetics. Oxford University Press, UK.

6. Mayo Clinic Staff Print. Brain AVM (arteriovenous Malformation). Mayo Clinic.

7. Alexander, Matthew D, Sander Connolly E, Meyers PM (2017) Symptoms of Vascular Malformations of the Brain. Brain \& Spine Foundation, ISBN 978-1-901-893-70-0.

8. Alexander MD, Connolly ES, Meyers PM (2010) Revisiting Normal Perfusion Pressure Breakthrough in Light of Hemorrhage-induced Vasospasm. World Journal of Radiology Baishideng 2(6): 230-232.

9. Pollock BE (2000) Occlusive Hyperemia: A Radiosurgical Phenomenon? Neurosurgery 47(5): 1178-1184.

10. Neurosurgical Consultants. Vascular Malformations: Arteriovenous Malformation. Neurosurgical Consultants, Dallas, USA.

\section{Your next submission with Juniper Publishers} will reach you the below assets

- Quality Editorial service

- Swift Peer Review

- Reprints availability

- E-prints Service

- Manuscript Podcast for convenient understanding

- Global attainment for your research

- Manuscript accessibility in different formats

( Pdf, E-pub, Full Text, Audio)

- Unceasing customer service

Track the below URL for one-step submission https://juniperpublishers.com/online-submission.php 